\title{
A Synopsis Model for Deterministic Behavioral Specifications of an Adaptable Agent
}

\author{
Dussadee Praserttitipong \\ Department of Computer Science, Faculty of Science, \\ Chiang Mai University, Chiang Mai, 50202, Thailand. \\ dussadee@science.cmu.ac.th \\ Peraphon Sophatsathit \\ Advanced Virtual and Intelligent Computing (AVIC) Center, \\ Faculty of Science, Chulalongkorn University, Bangkok, 10330, Thailand. \\ Peraphon.S@Chula.ac.th
}

\begin{abstract}
This paper proposes a deterministic behavioral approach that encompasses a service discovery model addressing service specifications and matchmaking processes. For service specifications, a synopsis of deterministic intention specification is nominated. Instead of using narrative description, the synopsis is formulated based on behavioral specifications and described by graphical representations to enable visual matching between service intention and advertised specifications. Moreover, an inequivalent matchmaking of this synopsis visualization model is also proposed as a means to investigate and assess various essential characteristic matchings between incomplete intention specification and complete service advertisement.
\end{abstract}

Keywords: $\quad$ service discovery, service specification, service advertisement, adaptable agent.

\section{Introduction}

Mobile Agent (MA) [3, 4] has emerged as realization of attempts to enable the adaptation under dynamic and unpredictable environments. This is, in part, because different hosts contain different resources. In order to model such an intelligent MA that can adapt itself to any foreign environments, all types of possible scenarios must be taken into account which unfortunately leads to an imploded MA.

To utilize an adaptation mechanism, one of the fundamental principles is to rely on service discovery technology. Currently, service discovery technology integrates researches on several communities [2, 14], such as reuse of Component-Based Software Engineering (CBSE), informa- tion retrieval of natural language service description, etc. There are two underlying processes in the proposed service discovery mechanism, i.e., service specification advertising and matchmaking process. The former is a service specification advertisement focuses on how to advertise the service, while the latter is a matchmaking process focuses on how to select the most suitable service.

There are many approaches for service discovery. The table-based service discovery approach $[9,10,12]$ is a conventional approach employed by most current standard service discovery technologies. The approach facilitates advertisement of services from both in-house libraries and thirdparty on-line repositories over the networks by considering fixed number of attribute valued pairs describing functional properties of the services. However, some inherent problems concerning with table-based service discovery persist. Because the matchmaking process relies on keywords matching, some services might be overlooked. There have been intensive researches addressing this problem by utilizing semantic concepts [8], ontology, and context-awareness $[1,5,6]$, which are regarded as successful principles for improving searching quality. Unfortunately, these studies have emphasized on modeling functional specifications, but less attention has been paid on behavioral specifications. Behavioral specifications are normally described in natural language. This in turn poses an obstacle to discovery as it remains unclear whether the service covers every facet of the user intentions. In contrast, deductive service discovery $[13,15]$ aims at expressing the service behavior based on formal specifications. Although, this approach offers formal descriptions for representing service behavior in a deterministic fashion, the matchmaking process constitutes high complexity and hard to use practically. In order to employ 
more practical matchmaking process, a software component matrix that represents formal specification is proposed in [11]. The logical form of a behavioral model refines the service capabilities using state transition diagrams, whereas the physical form is represented in matrix form. The matching process can thus be conducted by matrix manipulation operators.

Normally, in matchmaking process of the mentioned approaches, the process commences on case comparison between the request intention specification and the collected service advertisements. The most suitable service will be recommended to the requester. It is noticed that these approaches satisfy equivalent matchmaking between intention specification and service advertisement.

However, there are two significant issues in designing the MA. First, to move an MA over the network, the MA must be compacted to reduce transmission bandwidth. Second, since an MA works under dynamic and uncertain environments, the equivalent matchmaking cannot be satisfactorily determined.

The remaining of this paper is organized as follows. Section 2 describes the proposed approach. The experiment and evaluation is presented in Section 3. Section 4 concludes with future research direction.

\section{Proposed approach}

In this paper, an alternative service discovery approach is proposed, both on service specification and matchmaking process. For service specification, a deterministic synopsis intention specification is nominated in the proposed model. The model in turn addresses the bandwidth reduction issue as the desired behaviors of an MA are collected in a compact form. Moreover, synopsis visualization is introduced to formulate the specification by graphical representations. To cope with the second issue on dynamic and uncertain behavioral specification of an agent intention, some terminologies are established for subsequent derivations.

The agent intention specification is denoted by $\beta$, and the service advertisement is referred to as $\pi$. Both $\beta$ and $\pi$ are commonly called $\mathcal{I}$. Additionally, a set of concerning attributes for each object domain is defined as $\mathcal{A}=$ $\{\mathcal{A}(1), \mathcal{A}(2), \ldots, \mathcal{A}(\rho)\}$, where $\rho$ is the number of concerning attributes in each domain. Besides, the attribute values for any concerning attribute is defined as $\mathcal{A}(k)=\left\{a(k)^{1}, a(k)^{2}, \ldots, a(k)^{p_{k}}\right\}$, where $p_{k}$ is the number of concerning attribute values of $\mathcal{A}(k)$. Based on these definitions, it can be inferred that this model will not handle continuous attributes. Thus, the concerning attribute values need to be discretized beforehand.
Besides, a set of decision actions, denoted by $\Omega$, are categorized over a bipolar attitude scale as favorable class, FV Class, and unfavorable class, UFV Class. The definition of attitude is defined in [7] as " a predisposition that is learned in order to respond in a consistent way, either in favorable or unfavorable manner toward a specific object."

A number of overall attribute values, $n$, is a finite number for each domain of knowledge. $\alpha^{\beta}$ and $\alpha^{\pi}$ denote the number of conditional statements specified in $\beta$ and $\pi$, respectively. The variable $m$ is defined as the number of all possible conditional statements. Furthermore, the validity degree of reusing a service $\beta$ in place of $\pi$ is defined as a compatibility degree, denoted as $\Theta^{\beta, \pi}$.

Under certain environments whose resources are all known or adequately characterized in advance, all situations that the agent will encounter can be anticipated. In general, the agent intention specifications have a tendency to be the complete specification. Thus, compaction can be straightforwardly performed where the values of both $\alpha^{\beta}$ and $\alpha^{\pi}$ must be equal to $m$. This particular case is called the equivalent matchmaking. This quantitative measurement for the compatibility degree of reusing a service $\pi$ in place of $\beta$ can be derived from [11] as follows:

$$
\Theta^{\beta, \pi}=\frac{\left[\sum_{i=1}^{m} \Delta(\beta . \Omega(i), \pi \cdot \Omega(i))\right]}{m} \times 100
$$

In other words, $\Theta^{\beta, \pi}$ is proportioned to the similarity between $\beta . \Theta$ and $\pi . \Theta$. For conditional statement $i^{t h}$, the similarity of $\beta . \Theta(i)$ and $\pi . \Theta(i)$ can be assessed as follows:

$$
\Delta(\beta . \Omega(i), \pi . \Omega(i))= \begin{cases}1 & \text { if } \beta . \Omega(i) \equiv \pi \cdot \Omega(i) \\ 0 & \text { otherwise }\end{cases}
$$

where $\beta . \Omega(i) \equiv \pi . \Omega(i)$ if either $\beta . \Omega(i) \quad \in$ $F V$ and $\pi . \Omega(i) \in F V$ or $\beta . \Omega(i) \in U F V$ and $\pi . \Omega(i) \in$ $U F V . \Delta(\beta . \Omega(i), \pi . \Omega(i))$ will determine a value in the interval $[0: 1]$.

Conversely, dynamic and unpredictable environments notably differ over the certain environments whose resources are not all known or adequately characterized in advance. In this paper, the resources are referred to as the condition statements. The characteristics of the required services and the available services operating under this kind of environments may change based on contextual values with time. Therefore, when designing the agent, it is difficult to anticipate all complete situations the agent will encounter. In most cases, the agent intention specifications have a tendency to be incomplete. In contrast, service advertisements are mostly specified in form of the complete specifications. As such, the values of $\alpha^{\beta}$ and $\alpha^{\pi}$ are not equal. This case is noted as the inequivalent matchmaking. By applying 
Equation 1, this inequivalent matchmaking cannot be satisfactorily determined. There will be some indescribable situations over additional capabilities that may fall outside the boundary of intention specified in $\beta$.

\subsection{Service specification model}

\subsubsection{The synopsis model}

A synopsis of deterministic intention specification represents the characteristics of $\mathcal{I}$ ascribing the following definitions:

Definition 1 A synopsis of deterministic intention specification

Let $\mathcal{I}^{S Y}$ be a synopsis of deterministic intention specification of either $\beta$ or $\pi$. This specification can be modeled as a 2 -tuple $(\mathcal{E}, \mathcal{P})$, where $\mathcal{E}$ and $\mathcal{P}$ are $1 \times n$ specification features matrices.

$\mathcal{E}(j)$ is the entropy of an intention specification $\mathcal{I}$ for a concerning attribute value $j^{t h}$, that is,

$$
\mathcal{E}(j)=-\left(\left[\mathcal{P}(j) \times \log _{2} \mathcal{P}(j)\right]+\left[(1-\mathcal{P}(j)) \times \log _{2}(1-\mathcal{P}(j))\right]\right)
$$

$\mathcal{P}(j)$ is the probability of an intention specification $\mathcal{I}$ over the $F V$ class for a concerning attribute value $j^{\text {th }}$, i.e.,

$$
\mathcal{P}(j)=\frac{\text { number of } \mathcal{A}(j) \text { belonging to } F V \text { class }}{\text { number of occurrences } \mathcal{A}(j)}
$$

Given this definition, the ordinary intention specifications are arranged in standard form with equal size to overcome the limitations of the indescribable situations, whereby assessment of compatibility degree over the inequivalent match can be determined.

\subsubsection{Example}

In order to clarify the proposed model, a classical example from machine learning problem is given in Table 1.

For illustrative purpose based on the sample data in Table 1 , some instances of relevant elements $(\alpha=14)$ are demonstrated below.

- An attribute column vector $\mathcal{A}$ takes the form

$$
[\overbrace{\text { sunny overcast rain }}^{\text {outlook }} \overbrace{\text { hot mild cool }}^{\text {temperature }} \overbrace{\text { high normal }}^{\text {humidity }} \overbrace{\text { false true }}^{\text {windy }}]
$$

- An action class "FV Class" is an instance of one element in the set $\Omega$.
Table 1. The golf data set

\begin{tabular}{|l|l|l|l|l|l|}
\hline Cases & Outlook & Temperature & Humidity & Windy & Action Class \\
\hline$\alpha(1)$ & sunny & hot & high & false & UFV Class \\
\hline$\alpha(2)$ & sunny & hot & high & true & UFV Class \\
\hline$\alpha(3)$ & overcast & hot & high & false & FV Class \\
\hline$\alpha(4)$ & rain & mild & high & false & FV Class \\
\hline$\alpha(5)$ & rain & cool & normal & false & FV Class \\
\hline$\alpha(6)$ & rain & cool & normal & true & UFV Class \\
\hline$\alpha(7)$ & overcast & cool & normal & true & FV Class \\
\hline$\alpha(8)$ & sunny & mild & high & false & UFV Class \\
\hline$\alpha(9)$ & sunny & cool & normal & false & FV Class \\
\hline$\alpha(10)$ & rain & mild & normal & false & FV Class \\
\hline$\alpha(11)$ & sunny & mild & normal & true & FV Class \\
\hline$\alpha(12)$ & overcast & mild & high & true & FV Class \\
\hline$\alpha(13)$ & overcast & hot & normal & false & FV Class \\
\hline$\alpha(14)$ & rain & mild & high & true & UFV Class \\
\hline
\end{tabular}

- The number of overall attribute values can be calculated by $n=3+3+2+2=10$. Similarly, the number of all possible condition statements becomes $m=3 \times 3 \times 2 \times 2=36$.

- The probability of intention specification of one element in matrix $\mathcal{P}$, i.e., $\mathcal{P}(1)$, whose attribute value is "sunny" can be calculated based on Equation (4) as

$$
\mathcal{P}(1)=2 / 5=0.40
$$

Thus, the corresponding $\mathcal{E}(1)$ becomes

$$
\begin{aligned}
\mathcal{E}(1) & =-\left(\left[\mathcal{P}(1) \times \log _{2} \mathcal{P}(1)\right]+\left[(1-\mathcal{P}(1)) \times \log _{2}(1-\mathcal{P}(1))\right]\right) \\
& =-\left(\left[0.4 \times \log _{2}(0.4)\right]+\left[(1-0.4) \times \log _{2}(1-0.4)\right]\right) \\
& =0.97
\end{aligned}
$$

The $\mathcal{I}^{S Y}$ corresponding to the sample data in Table 1, comprises of matrix $\mathcal{P}$ and matrix $\mathcal{E}$

The matrix $\mathcal{P}$ takes the form

$\left(\begin{array}{llllllllll}0.40 & 1.00 & 0.60 & 0.50 & 0.67 & 0.75 & 0.43 & 0.86 & 0.75 & 0.50\end{array}\right)$

Similarly, the matrix $\mathcal{E}$ becomes

$\left(\begin{array}{llllllllll}0.97 & 0.00 & 0.97 & 1.00 & 0.92 & 0.81 & 0.99 & 0.59 & 0.81 & 1.00\end{array}\right)$

\subsubsection{A visualized model}

A visualized model of the specification synopsis is drawn in a 2-D shape, where the $\mathrm{x}$-axis accounts for the degree of entropy and the $y$-axis represents the probability of intention degree. 
Definition 2 A visualized model of a synopsis deterministic intention specification

Let $\mathcal{V}^{S Y}$ be a visualized model of a synopsis deterministic intention specification of either $\beta$ or $\pi$. This specification is modeled with vertices $(\mathcal{V})$ and edges $(\mathcal{G})$.

1. $\mathcal{V}(j)$ denotes an $\mathrm{x}-\mathrm{y}$ coordinate, $(\mathcal{E}(j), \mathcal{P}(j))$, of a concerning attribute value $j^{\text {th }}$.

2. $\mathcal{G}(j)$ denotes an edge from a vertex $\mathcal{V}(j)$ to a vertex $\mathcal{V}(j+1)$. The final $\mathcal{V}(n)$ wraps around to $\mathcal{V}(1)$, completing the representation cycle.

Figure 1 demonstrates three graphic examples of $\beta$ and $\pi$ on the left and right, respectively. As can be seen, the graphs depicting $\pi(1)$ in comparison with $\beta(1)$ are virtually no resemblance. The second one exhibits minor difference between $\beta(2)$ and $\pi(2)$. In contrast, the third graphs are strikingly similar.
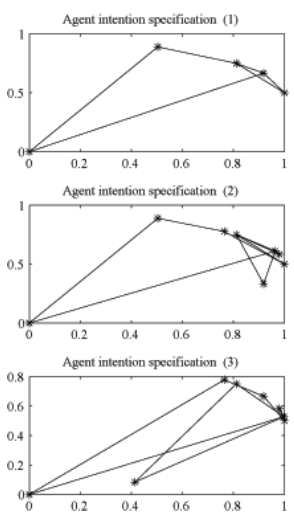
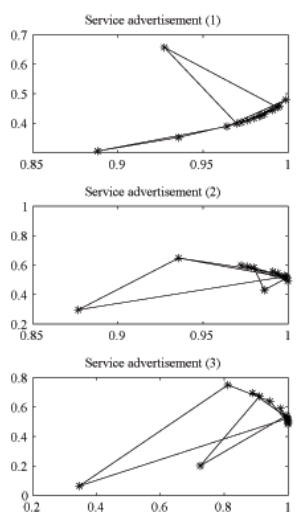

\section{Figure 1. Examples of visualized model}

The resulting compatible degrees of $\pi \mathrm{s}$ and $\beta \mathrm{s}$ are given as $31.71 \%, 70.60 \%$, and $89.81 \%$, respectively. The lowest compatibility degree is reported in the first instances as it is noticeable with the highest shape difference. On the other hand, the third example has the highest compatibility degree, where the graphs are very much alike in many ways.

This can be inferred that the compatible degree of $\pi$ comparing with $\beta$ is related to the similarity in shape of $\pi$ liken to $\beta$. Thus, the compatibility degree can somehow be calculated from the degree of characteristic preservation.

\subsection{A matchmaking process}

The ability for preserving the principal characteristics of $\beta$ is called a resemblance degree, which is quantified as follows:

$$
\Gamma^{\beta, \pi}=1 /\left[1+\nabla_{\mathcal{E}}^{\beta, \pi}\right]
$$

where $\nabla_{\mathcal{E}}^{\beta, \pi}$ is degree of discrepancy between the capabilities specified in $\beta$ and $\pi$. $\nabla_{\mathcal{E}}^{\beta, \pi}$ is given by

$$
\nabla_{\mathcal{E}}^{\beta, \pi}=\sum_{j=1}^{n}\left[\nabla_{\mathcal{E}}^{\beta, \pi}(j)\right]
$$

such that individual $\nabla_{\mathcal{E}}^{\beta, \pi}(j)$ can be determined from the following procedure:

$$
\begin{aligned}
& \text { if }\left|\mathcal{P}^{\beta}(j)-\mathcal{P}^{\pi}(j)\right|>0.4 \\
& \qquad \nabla_{\mathcal{E}}^{\beta, \pi}(j)=4.757 *\left|\mathcal{P}^{\beta}(j)-\mathcal{P}^{\pi}(j)\right|
\end{aligned}
$$

else

$$
\nabla_{\mathcal{E}}^{\beta, \pi}(j)=e^{\left[\mathcal{E}^{\beta}(j)-\mathcal{E}^{\pi}(j)\right]}
$$

Figure 2 demonstrates how matchmaking is performed visually. The value of $\nabla_{\mathcal{E}}^{\beta, \pi}$ of each element from the agent intention specification is matched with its corresponding element of service advertisement until all elements are exhausted.

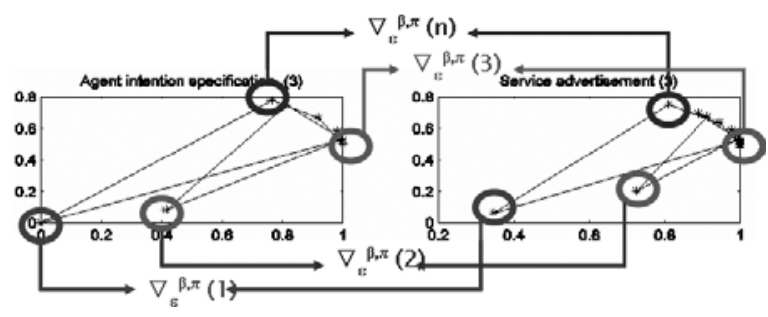

Figure 2. An example of visualized model

In order to confirm the correctness of the proposed concepts, statistical methods are chosen to validate the experiments. To measure the extent of characteristic preservation from the above matchmaking process, i.e., how close is the matchmaking accomplished, an assumption of the proposed characteristic preservation is defined as follows:

\section{If an agent executing its tasks by means of reusing service $\pi$ can preserve its principal characteris- tics declared in $\beta, \pi$ will be revealed as a com- patible service of $\beta$.}

The compatibility degree $\Theta^{\beta, \pi}$ is mapped as dependent variable, whereas $\nabla_{\mathcal{E}}^{\beta, \pi}$ is reduced to an independent variable. Regression and correlation analyses, namely, linear, quadratic, and cubic regression are employed. Mathematically, the linear, quadratic, and cubic equations can be expressed as in Equation (7), (8), and (9), respectively. 


$$
\begin{aligned}
& \Theta^{\beta, \pi}=\rho_{0}+\rho_{1} \nabla_{\mathcal{E}}^{\beta, \pi}+\epsilon \\
& \Theta^{\beta, \pi}=\rho_{0}+\rho_{1} \nabla_{\mathcal{E}}^{\beta, \pi}+\rho_{2}\left[\nabla_{\mathcal{E}}^{\beta, \pi}\right]^{2}+\epsilon \\
& \Theta^{\beta, \pi}=\rho_{0}+\rho_{1} \nabla_{\mathcal{E}}^{\beta, \pi}+\rho_{2}\left[\nabla_{\mathcal{E}}^{\beta, \pi}\right]^{2}+\rho_{3}\left[\nabla_{\mathcal{E}}^{\beta, \pi}\right]^{3}+\epsilon
\end{aligned}
$$

where $\rho_{0}, \rho_{1}, \rho_{2}$ and $\rho_{3}$ are regression coefficients.

There are two aspects that must be addressed in these analyses. The first aspect concerns with the nature of the relationship, where mathematical relation is used to predict the value of $\Theta^{\beta, \pi}$ from $\nabla_{\mathcal{E}}^{\beta, \pi}$. The second aspect concerns with the degree of the relationship, which refers to the strength of the relationship between $\Theta^{\beta, \pi}$ and $\nabla_{\mathcal{E}}^{\beta, \pi}$.

Additionally, hypothesis testing is applied to determine whether there exists an actual relationship between $\Theta^{\beta, \pi}$ and $\nabla_{\mathcal{E}}^{\beta, \pi}$. The basic hypotheses are defined as follows:

$$
\begin{aligned}
& H_{0}: \rho_{k}=0 \\
& H_{1}: \rho_{k} \neq 0
\end{aligned}
$$

where $\rho_{k}$ is the regression coefficient of order $k^{t h}$. For the linear, quadratic, and cubic relation testings, $\mathrm{k}$ is equal to 1,2 and 3 , respectively. If the null hypothesis $\left(H_{0}\right)$ holds true, it can be inferred that there exists a relationship between $\Theta^{\beta, \pi}$ and $\nabla_{\mathcal{E}}^{\beta, \pi}$. The contribution precipitating from these hypothesis testings is a mathematical model that can be used for coarse-gained estimation of the compatibility degree. Furthermore, the synopsis model is also verified whether it is an alternative for specifying the agent's behavior.

\section{Experiments and evaluation}

In this section, two main issues are experimented and evaluated, namely, hypothesis testing and contribution of the proposed model toward the indescribable situations. The experiments were carried out in two steps. First, $\mathcal{B}$ roker ${ }^{W B}$ conducts the validation process over the conventional white box specification. Second, B Broker ${ }^{S Y}$ conducts the validation process over a synopsis model. A concise example is given to demonstrate the issues under investigation. Procedural details are also presented in the sections that follow.

\subsection{Hypothesis testing and evaluation}

The equivalent matchmaking of complete agent intention specification and complete service advertisement was experimented under a controlled environment. The assumption describes the compatibility degree of $\beta$ and $\pi$, i.e., $\Theta^{\beta, \pi}$ in terms of the discrepancy degree $\nabla_{\mathcal{E}}^{\beta, \pi}$. The crucial issue is whether there is any relationship between $\Theta^{\beta, \pi}$ and $\nabla_{\mathcal{E}}^{\beta, \pi}$.
The results obtained from $\mathcal{B}$ roker ${ }^{W B}$ were noted as controlled results of this equivalent matchmaking. These results are subsequently employed in hypothesis testing. Mathematical evaluation formulated from the hypothesis testing step was carried out as the validation assessment procedure for $\mathcal{B r o k e r}^{S Y}$.

1. Hypothesis testing: The experiment performed over 1500 test cases where every test case was formulated as a complete intention specification. The F-statistic was applied on three regression analyses by $\mathcal{B}$ roker ${ }^{S Y}$ having significance rejecting level of $0.05 \%$. Figure 3 shows a comparison between the degree of compatibility and the degree of discrepancy. In the graph, the $\mathrm{x}$-axis represents the number of test cases, while the $y$-axis is the percentage of compatibility degree. The solid line depicts compatibility degree, whereas the dash line shows discrepancy degree. As can be seen, the discrepancy degree tends to be reversely related to the compatibility degree. The compatibility degree of the results increases as the degree of discrepancy decreases.

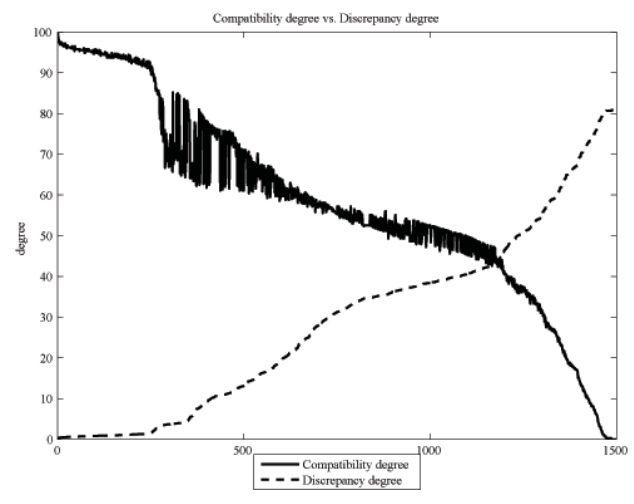

Figure 3. Comparison between the degree of compatibility and the degree of discrepancy

Table 2. Model Summary

\begin{tabular}{|c|c|c|c|c|c|c|}
\hline Equations & R-Square & standard error & F & df1 & df2 & Sig. \\
\hline Linear & 0.946 & 5.681 & 26438.095 & 1 & 1498 & 0.000 \\
\hline Quadratic & 0.946 & 5.682 & 13216.727 & 2 & 1497 & 0.000 \\
\hline Cubic & 0.961 & 4.875 & 12147.971 & 3 & 1496 & 0.000 \\
\hline
\end{tabular}

Table 2 summarizes significant values of 0.00 for all types of analyses. This implies that the null hypothesis is obviously rejected as the significance values are less than 0.05 . This can be inferred that there exists relationships between $\Theta^{\beta, \pi}$ and $\nabla_{\mathcal{E}}^{\beta, \pi}$. The R-square 
values are proportionated to the variations of $\Theta^{\beta, \pi}$ in the range of $94.6 \%, 94.6 \%$, and $96.1 \%$ for linear, quadratic, and cubic regression, respectively. The corresponding standard of errors are $5.681 \%, 5.682 \%$, and $4.875 \%$, respectively. This means that $\nabla_{\mathcal{E}}^{\beta, \pi}$ is a decisive factor influencing on the compatibility degree. Equations (7), (8), and (9) thus become

$$
\begin{aligned}
\Theta^{\beta, \pi}= & 88.264-1.055 \nabla_{\mathcal{E}}^{\beta, \pi} \\
\Theta^{\beta, \pi}= & 88.403-1.071 \nabla_{\mathcal{E}}^{\beta, \pi}+0.00022\left[\nabla_{\mathcal{E}}^{\beta, \pi}\right]^{2} \\
\Theta^{\beta, \pi}= & 91.764-1.985 \nabla_{\mathcal{E}}^{\beta, \pi}+0.033\left[\nabla_{\mathcal{E}}^{\beta, \pi}\right]^{2} \\
& -0.00029\left[\nabla_{\mathcal{E}}^{\beta, \pi}\right]^{3}
\end{aligned}
$$

\section{Validation assessment of the synopsis model:}

Figure 4 depicts the validation results obtained from both $\mathcal{B}_{\text {roker }}{ }^{W B}$, represented by dash lines, and $\mathcal{B}_{\text {roker }}{ }^{S Y}$, represented by solid lines. Applying the above three equations, the compatibility degree of the results acquired from $\mathcal{B}$ roker ${ }^{S Y}$ are almost the same as the compatibility degree of the results achieved from Broker $^{W B}$ in all types of analyses. Of the three analyses, the results from cubic fitting, shown in Figure 4(c), are most similar to the compatibility degrees comparing with the benchmark results. This indicates that cubic fitting is the most suitable regression model for representing the relationship between the compatibility degree and the discrepancy degree. The average accuracy degree of $\mathcal{B}$ roker ${ }^{S Y}$ with cubic fitting is $95.52 \%$, whilst the standard deviation is $4.74 \%$.
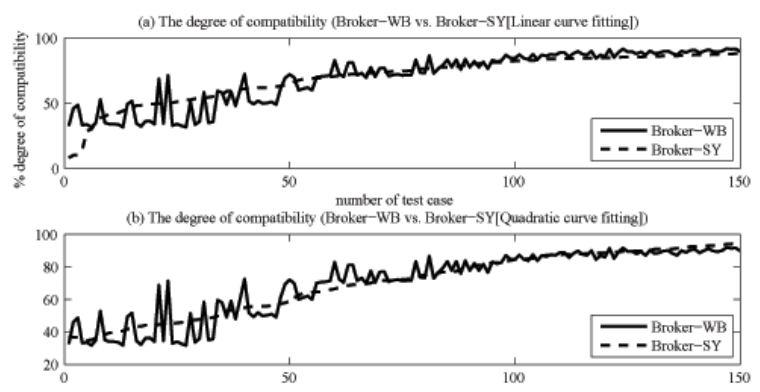

(c) The degree of compatibility (Broker-WB vs Broker-SY[Cubic curve fitting])

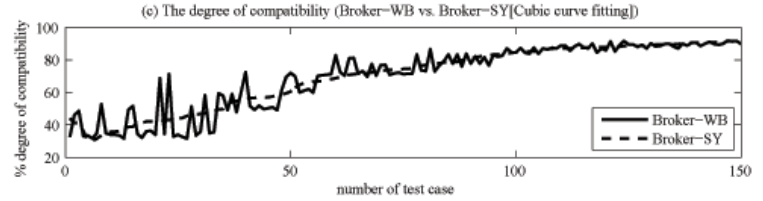

Figure 4. The compatibility degree

\subsection{Experimental results for the inequivalent match}

This experiment considers the matchmaking between incomplete agent intention specifications and complete service advertisements, having conditional statements (specification) $\alpha^{\beta}$ and $\alpha^{\pi}$ being not equal. Such matchmaking is categorized as inequivalent matchmaking. The controlling factors encompass two facets. First, all common attributes must be stated as concerning attributes of $\beta$ s with a random probability. In other words, all common attributes must relate to one or more conditions specified in each test case of $\beta$. Second, the agent's $i^{\text {th }}$ intention specification is a subset of the $i^{\text {th }}$ service advertisement. Thus, the assumption of this experiment can be noted as the results of compatibility degree between agent intention specifications and service advertisements which theoretically should be close to $100 \%$.

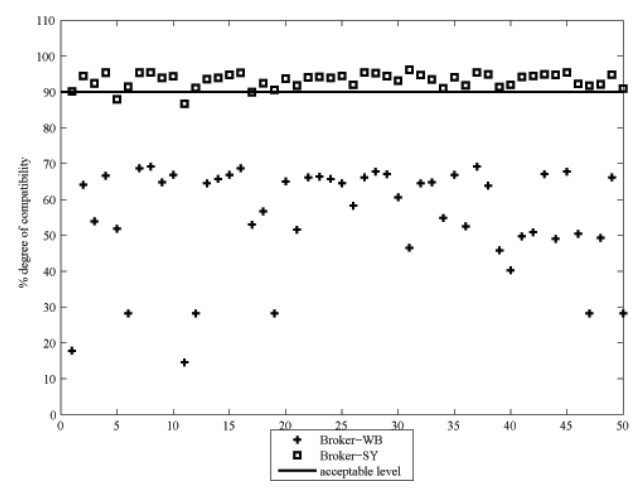

\section{Figure 5. The validation results from all Brokers}

Compatibility results obtained from Broker $^{W B}$ and Broker $^{S Y}$ over 50 cases of inequivalent matchmaking are shown in Figure 5, where the $\mathrm{x}$-axis denotes number of test cases and the $y$-axis is compatibility degree. The compatible degrees acquired from $\mathcal{B}$ roker ${ }^{W B}$ and $\mathcal{B}$ roker ${ }^{S Y}$ are $54.97 \%$ and $93.23 \%$, respectively. The standard deviation degrees are reported as $15.05 \%$ from Broker $^{W B}$ and $2.06 \%$ from Broker $^{S Y}$.

The solid line represents a predefined acceptable compatibility degree which is set to $90 \%$. The results from $\mathcal{B r o k e r}^{S Y}$ are two times higher than those from Broker $^{W B}$. Moreover, most test cases for $\pi \mathrm{s}$ are acceptable from reusing the service of $\beta$ s by $\mathcal{B r o k e r}^{S Y}$. These results satisfy the above assumption. The overall outcome of the proposed model employing $\mathcal{B r o k e r}^{S Y}$ attributes to the limitation of $\mathcal{B}$ roker ${ }^{W B}$ on inequivalent matchmaking. 


\section{Conclusions}

It is apparent that representing service function with loosely or lack of concern on service behavior leads to low qualitative values assessing over the matchmaking process. Similarly, overlooking of matchmaking over an inequivalent specification also brings about low qualitative values.

The proposed alternative service discovery model relies on, but not specified, the MA environments. The synopsis of deterministic intention specification is nominated. The proposed model compacts the deterministic agent intention specification that is suitable for inequivalent matchmaking process. Variation on representing service specification is introduced as the visualized model. The evaluation of this model is carried out with the help of statistical hypothesis testing.

The scope of this proposed model is restricted to coarsegrained validation because matchmaking results are computed from estimation process. Further investigation on fine-grained validation for improving the accuracy of results is a challenging research endeavor remained to be explored.

\section{References}

[1] G. D. Abowd, A. K. Dey, P. J. Brown, N. Davies, M. Smith, and P. Steggles. Towards a better understanding of context and context-awareness. In HUC, pages 304-307, 1999.

[2] A. Bernstein and M. Klein. Discovering services: Towards high-precision service retrieval. In WES, pages 260-276, 2002.

[3] R. Brandt and H. Reiser. Dynamic adaptation of mobile agents in heterogeneous environments. Journal of Communications and Networks, 3:307-316, December 2001.

[4] F. M. Brazier, B. J. Overeinder, M. van Steen, and N. J. Wijngaards. Agent Factory: Generative migration of mobile agents in heterogeneous environments, March 2002.

[5] T. Broens. Context-aware, ontology based, semantic service discovery, July 2004.

[6] C. Doulkeridis, E. Valavanis, and M. Vazirgiannis. Towards a context-aware service directory. In TES, pages 54-65, 2003.

[7] M. Fishbein and A. I. Belief, attitude, intention, and behavior: An introduction to theory and research. Addison-Wesley Publishing, Massachusetts, 1975.

[8] H.-M. Haav and T.-L. Lubi. A survey of concept-based information retrieval tools on the web. In Advances in Databases and Information Systems: Proceedings of 5th East-European Conference ADBIS'2001, pages 29-41, 2001.

[9] http://www.omg.org/technology/documents/corba_spec_catalog.htm. Object Management Group, Catalog of Corba/IIOP specifications (2004).

[10] http://www.uddi.org/pubs/Iru_UDDI_Technical_White_Paper.pdf. UDDI technical paper.

[11] S. Nakkrasae, P. Sophatsathit, and J. William R.Edwards. Fuzzy subtractive clustering based indexing approach for software components classification. International Journal of Computer \& Information Science, 5(1):63-72, March 2004.
[12] G. G. Richard. Service advertisement and discovery: Enabling universal device cooperation. IEEE Internet Computing, 4(5):18-26, 2000.

[13] K. Sycara, S. Widoff, M. Klusch, and J. Lu. Larks: Dynamic matchmaking among heterogeneous software agents in cyberspace. Autonomous Agents and Multi-Agent Systems, 5(3): 173-203, September 2002.

[14] Y. Wang and E. Stroulia. Semantic structure matching for assessing web-service similarity. In ICSOC, pages 194-207, 2003.

[15] A. M. Zaremski and J. M. Wing. Specification matching of software components. ACM Trans. Softw. Eng. Methodol., 6(4):333-369, 1997. 